\title{
Responsabilidad social en las secretarias de salud de la Guajira colombiana
}

\section{Social responsibility in the health offices of the colombian Guajira}

\author{
Sayuris Yelind Martínez Salas*, Cielo Margarita Rodríguez López", Danny Daniel López \\ Juvinao***
}

Universidad Rafael Belloso Chacín

Recibido: 12 de junio de 2020-Aceptado: 27 de agosto de 2021-Publicado: 1 de enero de 2022

Forma de citar este artículo en APA:

Martínez-Salas, S. Y, Rodríguez-López, C. M., \& López Juvinao, D. D. (2022). Responsabilidad social en las secretarias de salud de la Guajira colombiana. Revista Colombiana de Ciencias Sociales, 13(1), 173-191. https://doi.org/10.21501/22161201.3643

\section{Resumen}

La responsabilidad social es un concepto amplio donde se involucra la necesidad de responder a los grupos de interés con los que se interactúa; para el sector salud, este concepto es inseparable, ya que el Estado debe velar por hacer cumplir ciertos preceptos con el fin de garantizar la prestación de servicios en el territorio nacional, tomando en cuenta las diferencias y necesidades de cada uno de los municipios. Esta investigación tiene como propósito analizar la responsabilidad social en las secretarías de salud de la Guajira, Colombia. La investigación es descriptiva, de campo y no experimental. El instrumento fue la encuesta, misma que se aplicó a 58 personas de las secretarías de salud de 2 municipios de la Guajira colombiana. Se concluye que en el caso del Municipio Maicao no se tienen claros los elementos de la gestión de la

Doctora en ciencias mención gerencia, Universidad Rafael Belloso Chacín (URBE). Docente catedrática Universidad de La Guajira. Pertenece al grupo de investigación Emprendedores, Maicao, Colombia. Contacto: smartinezs@uniguajira.edu.co. ORCID: https://orcid.org/0000-0001-5348-3323

“" Doctora en ciencias mención gerencia, Universidad Rafael Belloso Chacín (URBE). Docente tiempo completo Universidad de La Guajira. Pertenece al grupo de investigación Emprendedores. Maicao, Colombia. Contacto: cmrodriguez@uniguajira.edu.co, ORCID: https://orcid.org/0000-0002-8383-5761, https://scholar.g0ogle.com/citations?user=blz08IMAAAAJ\&hl=es,

-.' Doctor en ciencias gerenciales, Universidad Rafael Belloso Chacín (URBE). Docente tiempo completo Universidad de La Guajira. Pertenece al grupo de investigación Ipaitug. Riohacha, Colombia. Contacto: dlopezj@uniguajira.edu.co, ORCID: http://orcid.org/0000-0002-9304-1105, https://scholar.google.es/citations?user=tBgckGkAAAJ\&hl=es 
responsabilidad social, aunque ponen en práctica algunos de los aspectos de la misma; mientras que en el municipio Uribia la gestión es deficiente, parecen desconocer el proceso, al mismo tiempo que los elementos no se toman en cuenta.

\section{Palabras clave}

Responsabilidad Social; Secretarías de Salud; Salud Pública; Colombia; Política de la salud.

\section{Abstract}

Social responsibility is a broad concept that involves the need to respond to the interest groups with which it interacts, for the health sector, this concept is inseparable, since the State must ensure that certain precepts are complied to guarantee the provision of services in the national territory, taking into account the differences and needs of each of the municipalities. The purpose of this research is to analyze social responsibility in the health network of La Guajira, Colombia. The research is descriptive, field and non-experimental. The instrument was the survey, it was applied to 58 people from the health secretary of two municipalities of the Colombian Guajira. It is concluded that in the case of the Maicao Municipality the elements of the management of social responsibility are not clear, but some the aspects of the same are put into practice. While management in the Uribia municipality is deficient, seemed of almost ignoring the process, at the same time that the elements are not taken into account.

\section{Keywords}

Social Responsibility; Health offices; Public health; Colombia; Health policy. 


\section{Introducción}

La responsabilidad social ocupa una posición estratégica mundial con respecto a la importancia de su temática. El origen de este concepto data de muchos años atrás, y siendo este un tema con tantas aristas, sería inexacto mencionar una fecha puntual del origen del concepto, sobre todo por los diversos sinónimos, interpretaciones, estrategias y conceptos relacionados que la han fortalecido convirtiéndola en una de las herramientas más fuertes con las que se cuenta en la sociedad para impulsar el desarrollo, hacer una gestión más eficiente y aplicar en cualquier escenario. Terán-Rosero et al. (2017) señalan que es a finales del siglo XX cuando alcanza su mayor relevancia en el ámbito empresarial, en especial relacionándolo con el término de sostenibilidad. Como señalan Martí-Noguera et al. (2017, p. 299) haciendo referencia a la responsabilidad social aplicada al ámbito universitario: "es actualmente un caleidoscopio en construcción y puede, o debiera, evolucionar a ser la reflexión-acción que ponga las bases de la sociedad inmersa en su cuarta revolución industrial", frase que puede ser extrapolada a otros sectores organizativos por conllevar un concepto de amplia aplicación.

Por tal razón, este concepto ha venido evolucionando desde la parte filantrópica, comprometiendo a todos los actores relacionados, hasta la evolución actual, donde más allá que una acción benéfica, explica la interacción y desarrollo sostenible de las organizaciones con su entorno sin perjudicar los grupos de interés. La responsabilidad social aporta desarrollo, debido a que las organizaciones deben asumir una actitud comprometida con la sociedad. Es decir, es una continua y permanente contribución que se hace de forma voluntaria para el mejoramiento de la sociedad, en todas sus dimensiones, en especial, las sociales, económicas y ambientales. Aunque, como señalan Tello-Castrillón y Rodríguez-Córdoba (2014), al principio se generaba confusión con respecto a si el término solo era aplicable a grandes corporaciones, posteriormente se observó que todo aquel sector que posea grupos de interés tiene inmerso el tema de la responsabilidad social, incluyendo a todos los sectores y tamaños; en este sentido, se traslada el término Responsabilidad Social Corporativa (RSC) por Responsabilidad Social Organizacional (RSO) para incluir a toda clase de organizaciones. En adelante solo se nombrará como responsabilidad social para evitar confusiones.

De esta manera "cualquier organización, empresa o no, tiene obligaciones sociales con sus empleados, proveedores, clientes, su comunidad y también con el nivel social" (Tello-Castrillón \& Rodríguez Córdoba, 2014, p. 124), lo que incluye todos los sectores de la sociedad, sobre todo aquellos relacionados con la atención de personas, como es el caso de salud.

El sector salud es de gran importancia para todos los países en virtud de lo que significa para la población, sobre todo hablando de la atención primaria en salud que, como señala Osores (2013), tiene que ver con asistencia sanitaria basada en métodos y tecnologías prácticas cientí- 
ficamente fundados, socialmente aceptables, al alcance de todos los individuos y familias de la comunidad. Según el informe anual, Atención Primaria en Salud: Avances y Retos en Colombia (Pineda Restrepo \& Jerez Trujillo, 2014), hay que mencionar que el gobierno nacional diseñó un Plan Decenal de Salud Pública (PDSP) 2012-2021 como un pacto social y mandato ciudadano con objetivos de mediano plazo, construido con la rectoría del Ministerio de Salud y Protección Social (2013a) en cumplimiento de la Ley 1438 (Congreso de la República, 2011), en donde se incluyen elementos de orden social, tomando en cuenta el impacto en las comunidades más allá del mero bienestar físico.

Terán-Rosero et al. (2017) señalan que hay pocos estudios en cuanto a la responsabilidad social en el sector salud, posiblemente porque se asume como un hecho por la propia naturaleza del sector; sin embargo, para el sector de la salud, en especial lo anterior es aplicable, tomando en consideración que los sistemas de salud son "sistemas sociales y culturales". Según Valor-Martínez y De la Cuesta-González (2005), la responsabilidad social se compone por obligaciones, compromisos legales y éticos, en el ámbito nacional e internacional con todos los grupos de interés de la organización. Dicho compromiso se deriva del impacto de las actividades u operaciones que se producen en el ámbito social, laboral, medioambiental y de los derechos humanos.

Teniendo en cuenta la definición de la Comisión Europea (2002), la responsabilidad social debe ser aplicada en colaboración cercana con los involucrados como un proceso que integre las preocupaciones sociales, medioambientales y éticas, respetando los derechos humanos, las preocupaciones de los consumidores, a fin de maximizar la creación de valor para sus propietarios, accionistas, partes interesadas y sociedad en sentido amplio.

Es importante enfatizar que existen autores como Ferrer (2013), que manifiesta que la responsabilidad social trae beneficios a las instituciones en los costos operativos, imagen de marca, reputación y acceso al capital. Sin embargo, en muchos casos existen prácticas deshonestas, desleales y fuera de la norma jurídica que están en las acciones empresariales. Para este autor, la responsabilidad social está enmarcada en la conducta, los valores y los principios de cada organización, de lo cual no escapan las organizaciones del sector salud.

Para Duque-Orozco et al. (2013), la gestión de la responsabilidad social es un modelo que sobrepasa el simple cumplimiento de las obligaciones legales, y que cada vez más organizaciones incorporan como factor de diferenciación y, por tanto, de supervivencia como parte del desarrollo en los mercados donde se desenvuelven. Por otra parte, en este punto, la responsabilidad no debe confundirse con la filantropía, porque la filantropía trata de promover la idea de desinterés al aplicarla. 
Por su parte, González y Márquez (2005) expresan que hay 2 elementos importantes para describir con respecto a las diversas interpretaciones del concepto: en primera instancia, el compromiso de las organizaciones, referido a la responsabilidad o compromiso de las empresas de operar agregando valor a la sociedad; en segundo lugar, la decisión voluntaria, ya que por lo general esta no es regulada por la ley.

De otro lado, Benjumea (2011) manifiesta que es una gestión de impactos (humanos, sociales y ambientales) que abarca a toda la organización y que debe ser realizada de manera ética e inteligente; por lo tanto, debe considerarse clave promover el acceso universal a los servicios de salud como esencial en la búsqueda del desarrollo sostenible, esto con el fin de asegurar que la salud sea un compromiso con las próximas generaciones.

En Colombia, sus actores no deben ser ajenos a este fenómeno, para avanzar hacia la equidad en salud y el desarrollo humano sostenible y sustentable, entendiendo, según Rivera-Hernández et al. (2017), que cuando se habla de sostenible tiene que ver con garantizar que el sistema productivo funcione y en cuanto a sustentable está relacionado con cuidar que los recursos que se usan no se agoten. La salud es uno de los elementos a cuidar para el logro del desarrollo de los pueblos, sobre todo porque las personas saludables tienen mayor capacidad para aprender, trabajar y contribuir a la economía y la sociedad. El acceso universal es un elemento clave en este aspecto. Por ello, debe tomarse en cuenta lo que señala De Ortúzar (2016), cuando resalta que "El ciudadano sólo no puede modificar su situación de salud, porque la misma depende de su entorno social y sanitario, y de las políticas públicas aplicadas para actuar sobre dicho entorno (Responsabilidad Social) (pp. 34-35)".

Indiscutiblemente, la salud es un catalizador para medir el impacto de las estrategias y políticas de desarrollo sostenible de un país. Puede actuar como un indicador de éxito, pues impulsa a otros sectores para que inviertan en ella, ya que es un indicador de progreso y una parte esencial del desarrollo social sostenible. Con el aporte, la salud pública puede ayudar a romper el círculo vicioso de la enfermedad y ofrecer apoyo para reducir las desigualdades dentro y entre las poblaciones. Como señala Soriano-Álvarez (2015, p. 293): “un concepto moderno de salud debe ir más allá de la atención médica individual o personalizada para abarcar dimensiones sociales más amplias como responsabilidad de todos".

Particularmente, en Colombia hay cambios que requieren un rediseño de los cuidados en salud para responder con más eficacia a las más nuevas y complejas necesidades, tales como la transición demográfica, la evolución tecnológica, el incremento de las expectativas sociales con respecto a la salud, al bienestar vital. Esos y muchos otros cambios requieren respuestas sanitarias, sobre todo piden una atención primaria de salud organizada y fuerte. 
Por su parte, el Ministerio de Salud y Protección Social (2013a) revela que los servicios de salud contribuyen solo en un $25 \%$, mientras que las circunstancias y condiciones del entorno donde se vive y se trabaja inciden en la aparición y desenlace de la enfermedad en un $65 \%$; por otro lado, los aspectos biológicos inciden en un $10 \%$. Así, se ha determinado que los servicios de salud en este país pueden considerarse necesarios, pero no suficientes para reducir las enfermedades y las muertes de las personas.

Según la Ley 1438 (Congreso de Colombia, 2011), la atención primaria en salud es la estrategia de coordinación intersectorial que permite la atención integral, desde la salud pública, la promoción de la salud, la prevención de la enfermedad, el diagnóstico, el tratamiento, la rehabilitación del paciente en todos los niveles de complejidad, a fin de garantizar un mayor nivel de bienestar en los usuarios, sin perjuicio de las competencias legales de cada uno de los actores del Sistema General de Seguridad Social en Salud de Colombia.

En los municipios de la Guajira colombiana, la dificultad de acceder a los centros de salud, las condiciones geográficas y el aumento considerable de población vulnerable -específicamente el grupo indígena wayuu-, así como las condiciones socioeconómicas, factores sociales, demográficos, entre otros aspectos relevantes -como las fuertes creencias culturales en relación con el concepto de salud y enfermedad arraigadas en las comunidades-, hacen que el concepto de salud se conciba desde otras perspectivas.

El desarrollo de esta investigación se llevó a cabo en los municipios de Maicao y Uribia; el primero está en el centro-este del Departamento de La Guajira. Se encuentra en un punto estratégico, convirtiéndose históricamente en un puente entre Colombia y Venezuela y una puerta hacia el intercambio comercial y cultural, posee una diversidad demográfica con habitantes de los pueblos indígenas wayuu y zenú; además de aglutinar una gran colonia de musulmanes procedentes de oriente medio, en su mayoría libaneses.

El segundo, Uribia, es un municipio colombiano al norte del Departamento de La Guajira. La mayoría de su población hace parte del pueblo wayuu, reconocido como propietario colectivo del gran resguardo indígena de la alta y media Guajira, ambos municipios tienen fronteras con la República Bolivariana de Venezuela, lo que hace posible que la población indígena del vecino país acceda a los servicios de salud de Colombia, debido a la cercanía de sus territorios y a que dentro de la cosmovisión e idiosincrasia del pueblo wayuu no existen fronteras entre estos dos países.

Se debe aclarar que, aunque el concepto de gestión de responsabilidad social puede estudiarse desde distintas perspectivas, en este caso se estudia a través del reconocimiento del código de conducta, estándares y declaración de principios, de manera que pueda observarse si dentro de la organización existe conocimiento acerca de la forma de comportarse, de medidas de desempeño y si están de acuerdo en los principios fundamentales a través de los cuales se implementa la res- 
ponsabilidad social. Al mismo tiempo, se aclara que los elementos a estudiar tienen que ver con voluntariedad, identidad, sostenibilidad y relación con grupos de interés, tomando en cuenta estos como esenciales para el caso del sector salud.

Con la aplicación de los lineamientos recientes relacionados al Decreto 1973 (Ministerio de Salud y Protección Social, 2013b) se ha impulsado la creación de un sistema indígena de salud propia e intercultural (SISPI), pues el enfoque principal del Plan de Decenal de Salud aún desconoce la incorporación sustancial de cosmovisiones étnicas en su diseño; por lo que, en vista de este interés del Estado en la creación de un sistema acorde a las diferencias territoriales, se analiza la responsabilidad social en las secretarías de salud de la Guajira, Colombia, específicamente en los Municipios Maicao y Uribia; ello permite examinar si llevan a cabo la gestión de responsabilidad social y si toman en cuenta sus elementos fundamentales, insumos que servirán para complementar la puesta en práctica del sistema indígena de salud y para conocimiento de los involucrados. A continuación, se presenta la metodología, los resultados, la discusión de estos y las conclusiones que se derivaron del estudio.

\section{Método}

Esta investigación es positivista con un método cuantitativo, de tipo descriptivo de campo, con diseño no experimental, transeccional o transversal (Hernández et al., 2014). Se escogió a la población de los municipios de Maicao y Uribia del Departamento de La Guajira, y específicamente al personal que desempeña actividades directamente relacionadas con la atención primaria en salud y que desarrollan sus funciones en los referidos municipios (Tabla 1). Se muestra en la siguiente tabla la cantidad de personal operativo, administrativo y de áreas afines (que están en otras áreas pero que participan dentro del proceso de atención primaria en salud).

\section{Tabla 1}

Características de la población

\begin{tabular}{lll} 
Secretarías de salud municipales de La Guajira & Secretaría de Salud de Maicao & Secretaría de Salud de Uribia \\
\hline Personal operativo & 29 & 14 \\
\hline Personal de otras áreas afines & 13 & 9 \\
\hline Personal administrativo & 17 & 12 \\
\hline Población & 59 & 35 \\
\hline Total & & 94 \\
\hline
\end{tabular}


Como se puede observar en la Tabla 1, la población objeto de estudio está representada por 59 funcionarios de la Secretaría de Salud del municipio de Maicao y 35 funcionarios de la Secretaria de Salud del municipio de Uribia, totalizando 94 sujetos.

Para Arias (2006), en este tipo de muestreo los elementos son escogidos con base en criterios o juicios preestablecidos por el investigador. En esta investigación, los criterios para seleccionar la muestra fueron: que los funcionarios tuvieran entre 1 y 2 años de servicio, que conocieran la cosmovisión de la cultura wayuu y que tuvieran conocimiento del Plan Decenal de Salud Públi$c a$. Para esto, se realizaron encuestas previas con estos elementos a todo el personal, en donde se pudo achicar la población de estudio, quedando representada por 58 sujetos (Tabla 2).

\section{Tabla 2}

Características de la muestra

\begin{tabular}{lccc}
$\begin{array}{c}\text { Secretarias de salud } \\
\text { municipales de La Guajira }\end{array}$ & $\begin{array}{c}\text { Secretaria de } \\
\text { Salud de Maicao }\end{array}$ & $\begin{array}{c}\text { Secretaría de } \\
\text { Salud de Uribia }\end{array}$ & TOTAL \\
\hline Personal operativo & 16 & 8 & 24 \\
\hline Personal de otras áreas afines & 10 & 5 & 15 \\
\hline Personal administrativo & 7 & 12 & 19 \\
\hline Población & 33 & 25 & 58 \\
\hline Total & & 58 & \\
\hline & Fuente: elaboración propia.
\end{tabular}

Para obtener los datos de la investigación se utiliza una encuesta (Ortegón, 2010). En cuanto a la confiabilidad del instrumento, se aplica la fórmula Alfa de Cronbach, obteniéndose una confiabilidad de 0.93; posteriormente a la confiabilidad se elabora una matriz de doble entrada, ubicando en la parte superior los ítems agrupados por bloques en atención a los indicadores, dimensiones y variables, y en el lado izquierdo los sujetos de la investigación, lo que arroja como resultado las frecuencias absolutas y relativas necesarias para el análisis y discusión, obteniendo así las respuestas a los objetivos planteados en esta investigación (Martínez, 2012).

Para analizar los resultados se tomó en cuenta el siguiente baremo:

Tabla 3

Baremo

\begin{tabular}{lclc}
\multicolumn{3}{c}{ Alternativas } & Valor \\
\hline Siempre & S & Excelente & 5 \\
Casi siempre & CS & Efectiva & 4 \\
A veces & AV & Regular & 3 \\
Casi nunca & CN & Deficiente & 2 \\
Nunca & & Pésimo & 1 \\
N & & & \\
\hline
\end{tabular}




\section{Resultados}

En este apartado se presentan los resultados con el propósito de darle respuesta al objetivo de análisis de la responsabilidad social en las secretarias de salud de los municipios de la Guajira colombiana; dichos resultados son producto de la aplicación del instrumento de recolección de datos aplicado al personal de las secretarías de salud municipales de La Guajira, específicamente las de Maicao y Uribia:

\section{Tabla 4}

Gestión de la responsabilidad social

\begin{tabular}{|c|c|c|c|c|c|c|c|}
\hline \multicolumn{8}{|c|}{ Municipio de Maicao } \\
\hline$n=33$ & \multicolumn{7}{|c|}{ Alternativas de respuesta } \\
\hline Indicador & S & CS & AV & $\mathrm{CN}$ & $\mathrm{N}$ & Media & $\begin{array}{c}\text { Desv. } \\
\text { tip. }\end{array}$ \\
\hline $\begin{array}{l}\text { Código de } \\
\text { conducta }\end{array}$ & 0 & 0 & $\begin{array}{l}21,21 \\
(\mathrm{fa}=7)\end{array}$ & $\begin{array}{c}42,42 \\
(\mathrm{fa}=14)\end{array}$ & $\begin{array}{c}36,36 \\
(\mathrm{fa}=12)\end{array}$ & 1,93 & ,96 \\
\hline Estándares & 0 & $\begin{array}{c}6,06 \\
(f a=2)\end{array}$ & $\begin{array}{c}45,45 \\
(f a=15)\end{array}$ & $\begin{array}{c}36,36 \\
(\mathrm{fa}=12)\end{array}$ & $\begin{array}{l}12,12 \\
(\mathrm{fa}=4)\end{array}$ & 2,33 & ,81 \\
\hline Declaración de principios & 0 & 0 & $\begin{array}{c}60,60 \\
(\mathrm{fa}=20)\end{array}$ & $\begin{array}{l}24,24 \\
(f a=8)\end{array}$ & $\begin{array}{l}15,15 \\
(f a=5)\end{array}$ & 3,51 & 1,39 \\
\hline Promedio general & 0 & 2,02 & 42,42 & 34,34 & 21,21 & 2,59 & 1,05 \\
\hline \multicolumn{8}{|c|}{ Municipio de Uribia } \\
\hline$n=25$ & \multicolumn{7}{|c|}{ Alternativas de respuesta } \\
\hline Indicador & $S$ & CS & AV & $\mathrm{CN}$ & $\mathrm{N}$ & Media & $\begin{array}{c}\text { Desv. } \\
\text { tip. }\end{array}$ \\
\hline $\begin{array}{l}\text { Código de } \\
\text { conducta }\end{array}$ & 0 & 0 & $\begin{array}{l}12,00 \\
(f a=3)\end{array}$ & $\begin{array}{c}52,00 \\
(f a=13)\end{array}$ & $\begin{array}{l}36,00 \\
(f a=9)\end{array}$ & 2,57 & 1,24 \\
\hline Estándares & 0 & 0 & $\begin{array}{l}28,00 \\
(\mathrm{fa}=7)\end{array}$ & $\begin{array}{c}56,00 \\
(\mathrm{fa}=14)\end{array}$ & $\begin{array}{l}16,00 \\
(\mathrm{fa}=4)\end{array}$ & 3,28 & 1,21 \\
\hline Declaración de principios & 0 & $\begin{array}{l}12,00 \\
(\mathrm{fa}=3)\end{array}$ & $\begin{array}{c}48,00 \\
(\mathrm{fa}=12)\end{array}$ & $\begin{array}{l}36,00 \\
(f a=9)\end{array}$ & $\begin{array}{c}4,00 \\
(\mathrm{fa}=1)\end{array}$ & 3,21 & 1,23 \\
\hline Promedio general & 0 & 4,00 & 29,33 & 48,00 & 18,66 & 3,02 & 1,22 \\
\hline
\end{tabular}

Discriminando los resultados de la Tabla 4 por indicadores, se percibió que el indicador $c o ́-$ digo de conducta arrojó $21,21 \%(\mathrm{fa}=7)$ en la alternativa "a veces" $(\mathrm{AV}) ; 42,42 \%(\mathrm{fa}=14)$ en la alternativa "casi nunca" $(\mathrm{CN})$ y $36,36 \%(\mathrm{fa}=12)$ en la opción "nunca" $(\mathrm{N})$, los cuales llevados al baremo en este orden traducen regular, deficiente y pésimo. Por su parte, el indicador estándares reporto $6,06 \%(\mathrm{fa}=2)$ en la opción "casi siempre" $(\mathrm{CS}) ; 45,45 \%(\mathrm{fa}=15)$ en la alternativa "a veces" (AV); 36,36 \% ( $(\mathrm{fa}=12)$ y 12,12 \% $(\mathrm{fa}=4)$ en la opción "casi nunca" $(\mathrm{CN})$, lo que traduce según el baremo como regular en su mayor porcentaje. El último indicador, pero no menos importante, declaración de principios, arrojó 60,60 \% en la alternativa "a veces" (AV) (fa=20); 24,24 $\%$ en "casi nunca" $(\mathrm{CN})(\mathrm{fa}=8)$ y $15,15 \%$ en "nunca" $(\mathrm{N})(\mathrm{fa}=5)$, lo que llevado al baremo indica que el mayor porcentaje se encuentra en regular. 
En cuanto al Municipio de Uribia se destaca que han sido medidos los mismos indicadores con un registro totalizado de 25 sujetos según la muestra abordada; allí las deducciones demuestran que el promedio general se registró en la categoría "casi nunca", con el $48 \%$, lo cual llevado al baremo indica que es deficiente el diagnóstico de gestión de la responsabilidad social en la atención primaria en salud, por lo cual se desconoce la situación actual del código de conducta, estándares y declaración de principios.

Asimismo, se extrajo la media y la desviación estándar a cada uno de los indicadores, quedando reflejado el promedio de la media en 3,02 y la desviación típica estándar 1,22. Al segregar la media y la desviación de los indicadores de la dimensión, los resultados arrojaron que el indicador código de conducta apuntó la media de 2,57 y la desviación típica de 1,24; el indicador estándares arrojó 3,28 en la media y 1,21 en la desviación estándar; el indicador declaración de principios reflejó 3,21 en la media y 1,23 en la desviación.

\section{Tabla 5}

Elementos de la responsabilidad social

\begin{tabular}{|c|c|c|c|c|c|c|c|c|}
\hline \multicolumn{9}{|c|}{ Municipio de Maicao } \\
\hline$n=33$ & \multicolumn{8}{|c|}{ Alternativas de respuesta } \\
\hline Indicador & \multicolumn{2}{|l|}{$S$} & CS & $\mathrm{AV}$ & $\mathrm{CN}$ & $\mathrm{N}$ & Media & $\begin{array}{l}\text { Desv. } \\
\text { tip. }\end{array}$ \\
\hline Voluntariedad & \multicolumn{2}{|l|}{$\begin{array}{l}3,03 \\
(\mathrm{fa}=1)\end{array}$} & $\begin{array}{l}15,15 \\
(\mathrm{fa}=5)\end{array}$ & $\begin{array}{l}42,42 \\
(f a=14)\end{array}$ & $\begin{array}{l}27,27 \\
(\mathrm{fa}=9)\end{array}$ & $\begin{array}{l}12,12 \\
(\mathrm{fa}=4)\end{array}$ & 2,57 & 1,24 \\
\hline Identidad & \multicolumn{2}{|l|}{$\begin{array}{l}9,09 \\
(\mathrm{fa}=3)\end{array}$} & $\begin{array}{l}6,06 \\
(\mathrm{fa}=2)\end{array}$ & $\begin{array}{l}48,48 \\
(\mathrm{fa}=16)\end{array}$ & $\begin{array}{l}27,27 \\
(\mathrm{fa}=9)\end{array}$ & $\begin{array}{l}9,09 \\
(f a=3)\end{array}$ & 4,28 & 1,21 \\
\hline Sostenibilidad & \multicolumn{2}{|l|}{0} & $\begin{array}{l}12,12 \\
(\mathrm{fa}=4)\end{array}$ & $\begin{array}{l}42,42 \\
(\mathrm{fa}=14)\end{array}$ & $\begin{array}{l}39,39 \\
(\mathrm{fa}=13)\end{array}$ & $\begin{array}{l}6,06 \\
(\mathrm{fa}=2)\end{array}$ & 4,21 & 1,23 \\
\hline $\begin{array}{l}\text { Relación con los grupos de } \\
\text { interés }\end{array}$ & \multicolumn{2}{|l|}{$\begin{array}{l}6,06 \\
(\mathrm{fa}=2)\end{array}$} & $\begin{array}{l}24,24 \\
(\mathrm{fa}=8)\end{array}$ & $\begin{array}{l}48,48 \\
(\mathrm{fa}=16)\end{array}$ & $\begin{array}{l}18,18 \\
(\mathrm{fa}=6)\end{array}$ & $\begin{array}{l}3,03 \\
(\mathrm{fa}=1)\end{array}$ & 3,33 & 1,61 \\
\hline Promedio general & \multicolumn{2}{|l|}{4,54} & 14,39 & 45,45 & 28,02 & 7,57 & 3,59 & 1,32 \\
\hline \multicolumn{9}{|c|}{ Municipio Uribia } \\
\hline \multicolumn{3}{|l|}{$n=25$} & \multicolumn{4}{|c|}{ Alternativas de respuesta } & & \\
\hline Indicador & $\mathrm{S}$ & CS & & AV & $\mathrm{CN}$ & $\mathrm{N}$ & Media & $\begin{array}{l}\text { Desv. } \\
\text { tip. }\end{array}$ \\
\hline Voluntariedad & 0 & $\begin{array}{l}12,00 \\
(\mathrm{fa}=3)\end{array}$ & & $\begin{array}{l}52,00 \\
(\mathrm{fa}=13)\end{array}$ & $\begin{array}{l}24,00 \\
(f a=6)\end{array}$ & $\begin{array}{l}12,00 \\
(\mathrm{fa}=3)\end{array}$ & 1,93 & ,96 \\
\hline Identidad & 0 & 0 & & $\begin{array}{l}28,00 \\
(\mathrm{fa}=7)\end{array}$ & $\begin{array}{l}56,00 \\
(\mathrm{fa}=14)\end{array}$ & $\begin{array}{l}16,00 \\
(\mathrm{fa}=4)\end{array}$ & 2,33 & ,81 \\
\hline Sostenibilidad & 0 & 0 & & $\begin{array}{l}32,00 \\
(\mathrm{fa}=8)\end{array}$ & $\begin{array}{l}56,00 \\
(f a=14)\end{array}$ & $\begin{array}{l}12,00 \\
(\mathrm{fa}=3)\end{array}$ & 3,51 & 1,39 \\
\hline $\begin{array}{l}\text { Relación con los grupos de } \\
\text { interés }\end{array}$ & 0 & 0 & & $\begin{array}{l}32,00 \\
(\mathrm{fa}=8)\end{array}$ & $\begin{array}{l}48,00 \\
(\mathrm{fa}=12)\end{array}$ & $\begin{array}{l}20,00 \\
(\mathrm{fa}=5)\end{array}$ & 2,12 & 1,10 \\
\hline Promedio general & 0 & 3,00 & & 36,00 & 46,00 & 15,00 & 2,47 & 1,06 \\
\hline
\end{tabular}


En cuanto a la Tabla 5, relacionada con los elementos de la responsabilidad social para la atención primaria en salud en los municipios de la Guajira colombiana, los resultados indican que en el Municipio de Maicao el promedio general repuntó con 45,45\% en la alternativa "a veces" (AV), lo cual, llevado al baremo, indica que la voluntariedad, la identidad, la sostenibilidad y la relación con los grupos de interés son los elementos de la responsabilidad social puestos en práctica de manera regular y no de forma excelente o efectiva.

Asimismo, se extrajo la media y la desviación estándar de cada uno de los indicadores, quedando reflejado el promedio de la media en 3,59 y la desviación típica estándar en 1,32. Al segregar la media y la desviación de los indicadores de la dimensión, los resultados arrojaron que el indicador voluntariedad arrojó 2,57 en la media y 1,24 en la desviación; identidad apuntó 4,28 en la media y 1,21 en la desviación estándar. Mientras el indicador sostenibilidad registró una media de 3,33 y una desviación de 1,61; el indicador relación con los grupos de interés reporto 3,33 en la media y 1,61 en la desviación típica.

Se acota que, en esta dimensión, todas las desviaciones estándar de los indicadores están por debajo del promedio de la media, indicando que esta muestra abordada está muy compacta alrededor de la media y ratificándose que en el Municipio de Maicao la voluntariedad, la identidad, la sostenibilidad y la relación con los grupos de interés son los elementos de la responsabilidad social puestos en práctica de manera regular.

De igual modo, se midió el indicador identidad, el cual reveló $9.09 \%(\mathrm{fa}=3)$ en la opción "siempre" (S); 6,06\% \% (fa=2); en la alternativa "casi siempre" (CS); 48,48 \% $(\mathrm{fa}=16)$ en la opción "a veces" (AV); 27,27 \%(fa=9) en la alternativa "casi nunca" (CN) y 9,09 (fa=3) en la opción "nunca" $(\mathrm{N})$, lo que llevado al baremo indica su mayor porcentaje en regular.

Otro indicador medido es la sostenibilidad, el cual arrojó 12,12\% ( fa=4) en la opción "casi siempre" (CS); 42,42 \% (fa=14) en la alternativa "a veces" (AV); 39,39 \% (fa=13) en la opción "casi nunca" (CN) y 6,06 (fa=2) en la alternativa "nunca" $(\mathrm{N})$, lo que se traduce como regular en su mayor porcentaje. Para concluir el análisis de la dimensión en el Municipio Maicao, se midió el indicador relación con los grupos de interés, el cual apuntó 6,06 (fa=2) en la opción "siempre" $(\mathrm{S}) ; 24,24 \%(\mathrm{fa}=8)$ en la alternativa "casi siempre" $(\mathrm{CS}) ; 48,48 \%(\mathrm{fa}=16)$ en la opción "a veces" $(\mathrm{AV}) ; 18,18 \%(\mathrm{fa}=6)$ en la alternativa "casi nunca" $(\mathrm{CN}) ;$ y 3,03 $(\mathrm{fa}=1)$ en la opción "nunca" $(\mathrm{N})$, lo que llevado al baremo indica regular.

De la misma forma, se midió la dimensión a través de estos indicadores en el Municipio Uribia, allí el promedio general se registró en la alternativa "casi nunca", indicando según el baremo que es deficiente la puesta en práctica de los elementos de la responsabilidad social para la atención primaria en salud en el municipio antes referido, porque no se toman en cuenta la voluntariedad, la identidad, ni la sostenibilidad y relación con los grupos de interés. 
Igualmente, se extrajo la media y la desviación estándar a cada uno de los indicadores, quedando reflejado el promedio de la media de 2,47 y 1,06 en la desviación típica estándar. Al segregar la media y la desviación de los indicadores de la dimensión, los resultados arrojaron que el indicador voluntariedad apuntó 1,93 en la media y ,96 en la desviación; identidad apuntó 2,33 en la media y ,81 en la desviación estándar; mientras el indicador sostenibilidad registró una media de 3,51 y una desviación de 1,39; y el indicador relación con los grupos de interés apuntó 2,12 en la media y 1,10 en la desviación típica.

Para concluir el análisis de esta dimensión en el municipio Uribia, se midió el indicador relación con los grupos de interés, el cual arrojó 32,00\% (fa. 8) en la alternativa "a veces" (AV); $48,00 \%$ (fa. 12) en la opción "casi nunca" $(\mathrm{CN})$; y $20 \%(\mathrm{fa}=5)$ en la alternativa "nunca" $(\mathrm{N})$, lo que llevado al baremo indica una aplicación deficiente.

Como puede observarse en el resumen de los datos arrojados, en la mayoría de los casos no existe una implementación adecuada de los elementos estudiados; sin embargo, el Municipio de Maicao muestra un mayor conocimiento e interés por los elementos relacionados con la responsabilidad social que el municipio de Uribia. Por ello, deben estudiarse las características particulares de cada uno para entender las dinámicas de implementación de los elementos estudiados.

\section{Discusión}

Aunque la responsabilidad social pareciera ser un tema aplicable a todos los sectores organizativos, e incluso en unos más que en otros, hay muchos casos, como en el de Briones et al. (2017), en que se demuestra que no se aplica y apenas se reconocen algunos de sus elementos.

Con respecto al diagnóstico de la gestión de la responsabilidad social en la atención primaria en salud en los municipios de la Guajira colombiana, los resultados evidencian que en el Municipio de Maicao, por lo menos de forma regular se reconocen los elementos de la gestión de la responsabilidad social en la atención primaria en salud, reconociendo de cierta forma la puesta en práctica del código de conducta, estándares y declaración de principios. Mientras que en el Municipio Uribia es deficiente el diagnóstico de gestión de la responsabilidad social en la atención primaria en salud, por lo cual se desconoce la situación actual del código de conducta, estándares y declaración de principios.

Lo que da respuesta a lo que señalan Terán-Rosero et al. (2017) cuando afirman que no hay muchos estudios al respecto a pesar de que no hay duda sobre la convergencia entre la naturaleza del servicio de salud con la responsabilidad social, tomando en cuenta aspectos culturales, éticos 
y ambientales, así como el necesario control social para lograr cobertura y calidad en el servicio a la sociedad. Se puede observar, entonces, que no se gestiona la responsabilidad social desde los conceptos básicos de su gestión.

Tales resultados se contradicen con lo que plasma el Libro Verde (Comisión Europea, 2002), donde se señala que la forma en que las organizaciones cumplen sus responsabilidades y llevan a cabo sus relaciones con los interlocutores difiere en función de aspectos sectoriales y culturales propios de cada uno. Primero, deben adoptar un código de conducta, estándares y declaración de principios; es decir, estos valores deben traducirse en medidas en toda la organización, desde las estrategias hasta las decisiones cotidianas. En el caso de estos municipios aún se encuentran atrasados por lo menos en el sector salud en cuanto al interés por la adopción de estos elementos, aunque no debe dejarse de lado que el Municipio de Maicao se encuentra más adelantado que Uribia, posiblemente por temas culturales.

Sobre los códigos de conducta, estos no se pueden considerar como únicos instrumentos válidos para el control social de las organizaciones, debido a que actualmente faltan incentivos atractivos en el mercado para que incorporen en su gestión los criterios de responsabilidad social aprobados en dichos códigos. El enfoque voluntarista en que se fundamentan estos códigos parte del supuesto que el mercado ofrece incentivos que llevarán a la organización a mejorar sus resultados sociales y medio ambientales. Sin embargo, la implementación de estos códigos dependerá mucho de la cultura de la región en la que se apliquen, como señalan Díaz de la Cruz y FernándezFernández (2016), resaltando que los valores sociales vienen dados por la cultura territorial; por tanto, la implementación de la responsabilidad social dependerá también del territorio y de los valores de la gente que lo habita.

Los estándares deben estar asociados al concepto de responsabilidad social, siendo los más apropiados los de rendición de cuentas y control social. Sin embargo, hay otro término usado: las normas. Estos estándares implican la consecución de una serie de resultados equivalentes para todas las organizaciones, algo inapropiado al concepto de responsabilidad social en relación a las diferencias significativas entre organizaciones.

En cuanto a los elementos de la responsabilidad social para la atención primaria en salud en los municipios de la Guajira colombiana, los resultados indican que en el Municipio de Maicao, la voluntariedad, la identidad, la sostenibilidad y la relación con los grupos de interés son los elementos de la responsabilidad social puestos en práctica de manera regular. Mientras en el Municipio de Uribia es deficiente la puesta en práctica de los elementos de la responsabilidad social para la atención primaria en salud porque no se toman en cuenta la voluntariedad, la identidad, ni la sostenibilidad y la relación con los grupos de interés. 
Este resultado es contradictorio con lo planteado por el Libro Verde (Comisión Europea, 2002), donde se define que existen elementos fundamentales para la creación del consenso en la responsabilidad social: voluntariedad, identidad, sostenibilidad y relación con los grupos de interés. Sobre la voluntariedad se revela que el desarrollo de las acciones debe ser voluntario, basándose en la idea de que las mismas son organizaciones que responden a criterios éticos de comportamiento que se transforman o modifican día a día en su interacción con el entorno, conformando una cultura ética empresarial adecuada a las realidades, exigencias y requerimientos de los grupos de interés.

Terán-Rosero et al. (2017) señalan que elementos como la voluntariedad son inherentes al término de responsabilidad social, pues sin una correcta gestión del trabajo voluntario sería imposible llevar a cabo una correcta gestión de la responsabilidad social.

En cuanto a la sostenibilidad es importante resaltar que, como lo señalan Tello-Castrillón y Rodríguez-Córdoba (2014):

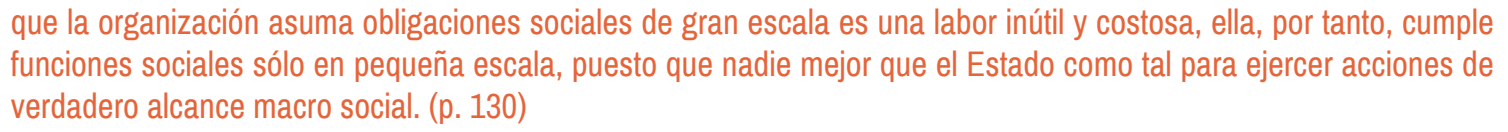

Finalmente, la relación con los grupos de interés son los cambios generados en el entorno de negocios al desplazar el enfoque tradicional de la organización basada en los accionistas (shareholders) por el enfoque hacia los grupos de interés (stakeholders), en el cual se asume que las organizaciones no rinden cuentas única y exclusivamente a sus accionistas, sino que también deben tomar decisiones considerando todos los actores sociales relacionados: empleados, proveedores, gobiernos nacionales y regionales, clientes, consumidores y organizaciones sociales, entre otros.

La forma de manejar los grupos de interés determina la aplicación de la responsabilidad social; como señalan Tello-Castrillón y Rodríguez-Córdoba (2014), “una organización pequeña podría ser socialmente más responsable que una gran organización si, por ejemplo, cobija y/o impacta más grupos de interés propios que los que cubre la segunda" (p. 131). En este caso, se observa que falta aún desarrollar este elemento para que pueda ser implementado de forma excelente.

Gutiérrez et al. (2006) señalan que Colombia es uno de los países de América Latina que más se ha preocupado por poner en práctica acciones de responsabilidad social, y esto se observa en el número de instituciones y fundaciones relacionadas con estos temas con las que se cuenta en el ámbito nacional, por lo cual la ventaja de tener una cultura de responsabilidad social beneficia su implementación. 
Se debe apuntar a lo que señalaba Volpentesta (2016) cuando concluye que se debe seguir apostando a "un cuerpo conceptual en el que en la racionalidad empresaria coexistan armónicamente el interés propio y el interés ajeno, la moral y las pautas de rendimiento eficienticista" (p. 212).

Por su parte, Vélez-Álvarez et al. (2018) demostraron en su estudio sobre la relación docenciaservicio en la formación de talento humano en salud, cómo se puede hacer visible la responsabilidad social integral a través de un conjunto de estrategias, por lo que en el sector salud la implementación de la responsabilidad social es casi que intrínseca y obligatoria.

En la discusión de resultados se resalta la importancia de la obtención de la respuesta de la encuesta como un diagnóstico en las secretarías de salud, ya que se puede observar cómo algunos estudios han demostrado la implementación de los elementos estudiados o cómo se ha corroborado la falta de implementación de estos. De manera que, al obtener una visión propia y comparar con estudios similares en el área, podremos entender la forma en que influencia el concepto en los diferentes sectores de la sociedad colombiana o de los países vecinos de Latinoamérica.

Es importante que se siga trabajando para que sectores fundamentales y de servicio a la comunidad sigan mejorando sus servicios; para eso, la investigación en las universidades es fundamental, ya que puede, como en este caso, mostrar las debilidades que se encuentran presentes al verificar la aplicación de conceptos que quizás parezcan comunes en el habla cotidiana de las instituciones gubernamentales. Un gran apoyo es, entonces, la investigación de campo desde las universidades para verificar la forma a través de la cual se implementan los conceptos que se señalan.

\section{Conclusiones}

La responsabilidad social en el sector salud de la Guajira Colombiana (específicamente en los municipios de Maicao y Uribia) no se implementa, el reconocimiento de algunos de los términos fundamentales de su gestión y los elementos que la conforman no dan suficientes bases para decir que se haya hecho. Se observa cómo el personal relacionado a las secretarías de salud puede tener intención de reconocer la importancia de este proceso, sin embargo, su implementación es regular o deficiente tomando en cuenta las respuestas obtenidas de la encuesta.

La responsabilidad social es un tema complejo y de gran importancia, sobre todo en el sector salud, en donde la relación entre los grupos de interés demanda la mejora continua de los servicios para garantizar los derechos fundamentales de la población. Sin embargo, no puede dejarse 
de lado la cultura propia de cada una de las regiones, que determina de cierta forma el éxito o fracaso de la implementación de políticas gubernamentales relacionadas con la responsabilidad social.

Siendo la atención primaria en salud una arista del sector salud que se vuelve fundamental en la cotidianidad del ciudadano, se hace necesario reconocer el beneficio que puede obtenerse de la implementación de la gestión de la responsabilidad social como elemento de conjunción entre los distintos grupos de interés involucrados, que a mediano y largo plazo traerá beneficios al proceso de atención al ciudadano y a todos los procesos asociados.

Se reconoce, entonces, que el municipio Maicao se encuentra con alguna ventaja ante el reconocimiento de ciertos elementos respecto del Municipio Uribia, por lo que pudiera iniciarse un trabajo de implementación piloto en el que se verifique cómo pudiesen verse beneficiados con elementos como el impulso de la voluntariedad y la relación con los grupos de interés, de manera que puedan observarse los beneficios que esto aportaría al sector y a la población en general. Luego de verse los beneficios puede seguirse ampliando al resto de los municipios cercanos.

Algunos de los lineamientos iniciales para esta implementación piloto pueden ser:

- Revisar los elementos que funcionan en la implementación de responsabilidad social en el Municipio de Maicao.

- Crear planes de adiestramiento para educar a los municipios cercanos respecto de las actividades realizadas, mostrando los resultados obtenidos (ejemplo en temas de voluntariedad y relación con grupos de interés).

- Revisar y evaluar continuamente las actividades relacionadas implementadas para registrar los resultados obtenidos en el proceso.

- Crear un plan propio en función de las acciones resultantes.

Este estudio, además, da pie a seguir investigando al respecto de temas como la gobernanza interorganizacional y el gobierno corporativo en el caso del sector salud, ya que la responsabilidad social está relacionada directamente con estos aspectos.

\section{Conflicto de intereses}

Los autores declaran la inexistencia de conflicto de interés con institución o asociación comercial de cualquier índole. 


\section{Referencias}

Arias, F. (2006). El proyecto de investigación introducción a la metodología científica. 6.ta Edición. Episteme.

Benjumea, J. (2011). Prácticas de responsabilidad social empresarial que ostentan las universidades públicas y privadas de la ciudad de Manizales (Tesis de Maestría, Universidad Nacional). https://repositorio.unal.edu.co/handle/unal/8161

Briones, V., Torres, C., Rojas, S., Jiménez, J., \& Ávila, M. (2017). Análisis de la Responsabilidad Social Empresarial del Sector de Confecciones Pymes de Guayaquil. Revista Espacios, 38(31), s.p. https://www.revistaespacios.com/a17v38n31/a17v38n31p09.pdf

Comisión Europea. (2002). Libro Verde de la Comisión Europea. Fomentar un marco europeo para la responsabilidad social de las empresas. ESADE. http://itemsweb.esade.edu/wi/ research/iis/pdfs_web/Libro_Verde.pdf

Congreso de Colombia. (2011). Ley 1438 de 2011. Por medio la cual se reforma el sistema general de seguridad social en salud. https://www.minsalud.gov.co/Normatividad_Nuevo/ LEY\%201438\%20DE\%202011.pdf

De Ortúzar, M. (2016). Responsabilidad social vs. responsabilidad individual en salud. Revista de Bioética y Derecho, (36), 23-36. https://dx.doi.org/10.1344/rbd2016.36.15374

Díaz de la Cruz, C., \& Fernández-Fernández, J. (2016). Marco conceptual de la ética y la responsabilidad social empresarial: un enfoque antropológico y estratégico. Revista Empresa y Humanismo, XIX(2), 69-118. https://doi.org/10.15581/015.XIX.2.69-118

Duque-Orozco, Y., Cardona-Acevedo, M., \& Rendón-Acevedo, J. (2013). Responsabilidad social empresarial: Teorías, índices, estándares y certificaciones. Cuadernos de Administración y Negocios, 29(50), 196-206. http://www.scielo.org.co/pdf/cuadm/v29n50/v29n50a09.pdf

Ferrer, J. (2013). La dimensión externa de la responsabilidad social empresarial de CANTV. Telos: Revistas de Estudios Interdisciplinarios en Ciencias Sociales, 15(3), 388-399. http:// ojs.urbe.edu/index.php/telos/article/view/2185/2035

González, R., \& Márquez, P. (2005). El dilema entre la responsabilidad social y rentabilidad empresarial. Debates IESA, X(3), 65-69. 
Gutiérrez, R., Avella, L. F., \& Villar, R. (2006). Aportes y desafios de la responsabilidad social empresarial en Colombia. Fundación Corona.

Hernández, R., Fernández, C., \& Baptista, P. (2014). Metodología de la investigación. McGraw Hill.

Martí-Noguera, J. J., Puerta-Lopera, I. C., \& Rojas-Román, P. (2017). A propósito de la Responsabilidad Social Universitaria [Editorial]. Revista Colombiana de Ciencias Sociales, 8(2), 294-301. http://dx.doi.org/10.21501/22161201.2291

Martínez, C. (2012). Estadística y muestreo. Ecoe.

Ministerio de Salud y Protección Social (2013a). Plan Decenal de Salud Pública PDSP, 20122012, La salud en Colombia la construyes tú. MinSalud.

Ministerio de Salud y Protección Social (2013b). Decreto 1973. Por el cual se crea la Subcomisión de Salud en la Mesa Permanente de Concertación con los Pueblos y Organizaciones Indigenas. https://bit.ly/3ED4GUY

Ortegón, M. (2010). Estadística descriptiva. Universidad Nacional Abierta y a Distancia.

Pineda Restrepo, B, \& Jerez Trujillo, A. (2014). Informe Anual 2014. Atención Primaria en Salud: avances y retos en Colombia. Programa: Así vamos en Salud.

Osores, P. (2013). Atención a poblaciones con exclusión social: experiencias y reflexiones. Revista Ciencias Salud, 4(1), 83-86. https://www.semanticscholar.org/paper/Atenci\%C3\%B3na-poblaciones-con-exclusi\%C3\%B3n-social\%3A-y-Tello-P\%C3\%A1vel/0f933a4e4a60e 32e15606b9f9e9b20f02458e279

Rivera-Hernández, J. E., Blanco-Orozco, N. V., Alcántara-Salinas, G., Houbron, E. P., \& PérezSato, J. A. (2017). ¿Desarrollo sostenible o sustentable? La controversia de un concepto. Posgrado y Sociedad Revista Electrónica del Sistema de Estudios de Posgrado, 15(1), 57-67. https://doi.org/10.22458/rpys.v15i1.1825

Soriano-Álvarez, C. (2015). Las sociedades científicas y la responsabilidad social en salud [editorial]. Revista de Gastroenterología del Perú, 35(4), 293. http://www.revistagastroperu. com/index.php/rgp/article/viewFile/92/90

Tello-Castrillón, C., \& Rodríguez-Córdoba, M. (2014). Categorías conceptuales para el estudio de la responsabilidad social organizacional. Hallazgos, 11(22), 119-135. https://doi. org/10.15332/s1794-3841.2014.0022.07 
Terán-Rosero, G., Montenegro-Obando, B., Bastidas-Guerrón, J., Realpe-Cabrera, I., VillarrealSalazar, F., \& Fernández-Lorenzo, A. (2017). Análisis crítico de la responsabilidad social en entidades de salud. Revista Cubana de Investigaciones Biomédicas, 36(1), 1-10. http:// scielo.sld.cu/scielo.php?script=sci_arttext\&pid=S0864-03002017000100020

Valor-Martínez, C., \& De la Cuesta-González, M. (2005). Efectividad de los códigos para el control social de la empresa. CIRIEC-España, Revista de Economía Pública, Social y Cooperativa, (53), 163-190. https://www.redalyc.org/pdf/174/17405312.pdf

Vélez-Álvarez, C., Jaramillo-Ángel, C., \& Giraldo-Osorio, A. (2018). Docencia-servicio: responsabilidad social en la formación del talento humano en salud en Colombia. Educación Médica, 19(S2), 179-186. https://doi.org/10.1016/j.edumed.2017.08.002

Volpentesta, J. (2016). Tendencia y perspectiva de la responsabilidad social empresaria. Revista Cientifica "Visión de Futuro", 20(2), 193-215. https://bit.ly/3nSsoqf 